\title{
Camshaft Loosening Diagnosis on the Basis of Generalised Force Recognition at the Centre of Gravity of an Engine
}

\author{
Chuanyan Xu $\mathbb{D}^{1},{ }^{1}$ Lixue Meng, ${ }^{1}$ Ruyan Gong, ${ }^{2}$ Xun Gong, ${ }^{1}$ and Aijuan $\mathrm{Li}^{1}$ \\ ${ }^{1}$ School of Automotive Engineering, Shandong Jiaotong University, Jinan 250023, China \\ ${ }^{2}$ Weihai Institute of Metrology and Inspection for Product Quality Standards, Weihai 264200, China \\ Correspondence should be addressed to Chuanyan Xu; 254883652@qq.com
}

Received 22 June 2021; Accepted 12 August 2021; Published 20 August 2021

Academic Editor: Anil Kumar

Copyright (C) 2021 Chuanyan Xu et al. This is an open access article distributed under the Creative Commons Attribution License, which permits unrestricted use, distribution, and reproduction in any medium, provided the original work is properly cited.

A valve mechanism supports the working process of an engine cylinder, and a camshaft is a key component required to open and close a valve. When a camshaft loosens, the balance of the engine disrupts. In the meanwhile, the generalised force at its centre of gravity (CG) alters. This study proposed a novel technique to detect camshaft loosening based on recognising the generalised force at the CG of the engine. We conducted Hanning windowed interpolation of discrete spectra to extract the precise phase and amplitude by utilising the acceleration signals at the engine cylinder and mounts and cylinder head. We then accurately computed the generalised force at the CG. Finally, we accurately extracted the camshaft loosening features by analysing the main harmonic orders for the generalised force. As indicated by simulations, our method can be used to effectively detect combustion engine faults involving camshaft loosening.

\section{Introduction}

A camshaft is a key component required to open and close the valve in an engine valve distribution mechanism. Camshaft projections are in contact with a tappet. Excessive stress due to contact between the camshaft projection and the tappet roller may cause wear failure [1]. Additionally, under long service time, a camshaft is subject to alternating load torque, bending moment, and impact load. It possibly operates with an angular vibration along with a bending vibration. Bearing and bush wear and camshaft loosening can lead to overall wear and unpleasant noise. The efficiency and reliability of camshafts decrease because of inaccurate movement, eventually resulting in force variations at the centre of gravity (CG). An injection pump camshaft of a truck diesel engine failed after a mere $13,000 \mathrm{~km}$ run because fatigue cracks were initiated at the keyway root through circumferential tangential stress because the stationary frictional force between the camshaft cone and timer working on keyway edges was inadequate [2]. A diesel engine camshaft underwent instantaneous circumferential cracking that propagated brittlely due to tensile stress generated at the site of camshaft straightening [3].
Du and $\mathrm{Yu}$ [4] developed an engine power assembly and accompanying valve train. They compared the vibration acceleration signals of the engine cylinder head in normal and fault states to determine the loosening fault features of camshaft bearings. Diagnosis using mechanical systems is a key subject in modern industry. In recent years, intelligent methods have been widely used in structural diagnosis to detect faults in 3D printers. Li et al. introduced an extremelearning-machine-based intelligent solution for fault diagnosis that used a low-cost and precise attitude sensor and helped diagnostic measure studies for determining faults in delta 3D printers $[5,6]$. Kumar et al. developed a diagnostic technique based on deep learning to recognise defects in two-wheeler vehicle engines [7]. Ting et al. [8] determined the cluster centre in a radial basis function neural network by using the $K$-means clustering algorithm to study faults in camshaft grinders. Fault samples are critical to initiate an artificial intelligence (AI) mode. However, achieving convenient and thorough sampling in actual applications is difficult because mostly mechanical systems are healthy and normal. Thus, it becomes difficult to attain AI model training even with sufficient fault samples [9]. 
The diagnosis of camshaft faults must be effectual and accurate to ensure that the engine operates as usual [10]. Vibration signals, containing a large amount of fault information, are extensively utilised to diagnose faults [11-14]. The vibration pattern of an engine can offer diverse information about its state. In most cases, engine faults are manifested directly as the fluctuation of generalised force at the engine CG, achieved by utilising the acceleration signals at the engine mounts. Xu et al. computed generalised force at the engine CG and extracted features to detect single and continuous/intermittent double cylinder misfires [15]. The primary vertical force for a four-cylinder four-stroke motor refers to the second-order force, and the half- and first-order forces are nearly zero [16]. However, the force at the CG varies with camshaft faults.

Our study proposes a novel technique to detect camshaft loosening based on recognising the generalised force at the engine CG. We extracted the precise phase, frequency, and amplitude through Hanning windowed interpolation. Two prime advantages of our detection method are its high fault sensitivity and less subjectivity to external factors and experimental conditions.

The following presents the organization of the rest of the paper. Section 2 introduces the identification principles for generalised force at the CG. Section 3 describes the diagnostic procedure for camshaft loosening based on the computed generalised force and illustrates the simulation results. The conclusions are made in Section 4.

\section{Theoretical Fundaments}

2.1. Generalised Force Estimation Method. As shown in Figure 1, a powertrain mounting system is simplified into a six-degree-of-freedom vibration model to calculate the engine excitation force.

Assuming a slight movement of the engine mount system, the kinematic equation for the power assembly mounting system is

$$
M \ddot{Q}(t)+C \dot{Q}(t)+K Q(t)=F(t),
$$

where $M$ denotes the $6 \times 6$ rigid mass matrix of the engine, $C$ denotes the $6 \times 6$ damping matrix, $K$ denotes the $6 \times 6$ stiffness matrix, and $Q$ and $F$ are the generalised displacements at the engine CG and $6 \times 1$ generalised force vector, respectively.

$$
\underset{6 \times 6}{M}=\left[\begin{array}{cccccc}
m & 0 & 0 & 0 & m z_{c} & -m y_{c} \\
0 & m & 0 & -m z_{c} & 0 & m x_{c} \\
0 & 0 & m & m y_{c} & -m x_{c} & 0 \\
0 & -m z_{c} & m y_{c} & J_{x x o} & -J_{x y o} & -J_{x z o} \\
m z_{c} & 0 & -m x_{c} & -J_{x y o} & J_{y y o} & -J_{y z o} \\
-m y_{c} & m x_{c} & 0 & -J_{x z o} & -J_{y z o} & J_{z z o}
\end{array}\right],
$$

where $m$ is the engine mass; $x_{c}, y_{c}$, and $z_{c}$ are the CG coordinates in the OXYZ reference system; $J_{x x o}, J_{y y o}$, and $J_{z z o}$ are the inertial moments for $X, Y, Z$ coordinates, separately; and $J_{x y o}, J_{y z o}$, and $J_{x z o}$ are the cross inertial moments.

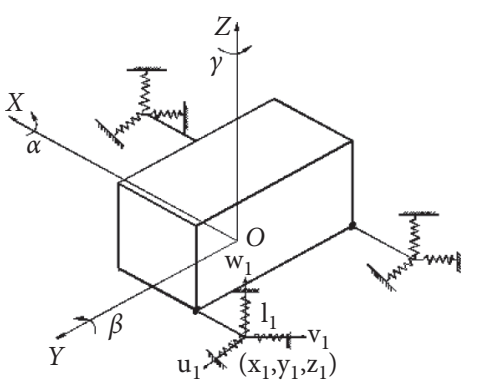

FIgURE 1: Simplified model of a powertrain.

$$
\underset{6 \times 6}{K}=\left[\begin{array}{llllll}
k_{x x} & k_{x y} & k_{x z} & k_{x \alpha} & k_{x \beta} & k_{x \gamma} \\
k_{y x} & k_{y y} & k_{y z} & k_{y \alpha} & k_{y \beta} & k_{y \gamma} \\
k_{z x} & k_{z y} & k_{z z} & k_{z \alpha} & k_{z \beta} & k_{z \gamma} \\
k_{\alpha x} & k_{\alpha y} & k_{\alpha z} & k_{\alpha \alpha} & k_{\alpha \beta} & k_{\alpha \gamma} \\
k_{\beta x} & k_{\beta y} & k_{\beta z} & k_{\beta \alpha} & k_{\beta \beta} & k_{\beta \gamma} \\
k_{\gamma x} & k_{\gamma y} & k_{\gamma z} & k_{\gamma \alpha} & k_{\gamma \beta} & k_{\gamma \gamma}
\end{array}\right],
$$

where $k_{x x}, k_{y y}$, and $k_{z z}$ are the total reciprocating stiffnesses of elastic support; $k_{x y}, k_{x z}$, and $k_{z y}$ are the coupling stiffnesses of elastic support; and $k_{\alpha \alpha}, k_{\beta \beta}$, and $k_{\gamma \gamma}$ are the rotary stiffnesses about the coordinate axis.

$$
\begin{array}{r}
\underset{6 \times 1}{F}=\left\{\begin{array}{c}
F_{x} \\
F_{y} \\
F_{z} \\
M_{x} \\
M_{y} \\
M_{z}
\end{array}\right\}, \\
\underset{6 \times 1}{Q}=\left\{\begin{array}{c}
X_{o} \\
Y_{o} \\
Z_{o} \\
\alpha \\
\beta \\
\gamma
\end{array}\right\},
\end{array}
$$

where $F_{x}, F_{y}$, and $F_{z}$ represent transitional applied forces; $M_{x}, M_{y}$, and $M_{z}$ represent the applied moments about point $O ; X_{o}, Y_{o}$, and $Z_{o}$ are point $O^{\prime} s$ transitional displacements; and $\alpha, \beta$, and $\gamma$ are point $O^{\prime} s$ rotational displacements.

Using Fourier transformation on the two sides of equation (1), we derive

$$
\left[M-\frac{K}{(2 \pi f)^{2}}-\frac{j C}{2 \pi f}\right] \ddot{Q}(f)=F(f) .
$$

Considering $s$ points at which acceleration can be determined, where the coordinates of the $i$ th point $(i=1, \ldots, s)$ for the CG are $\left[\begin{array}{lll}x_{i} & y_{i} & z_{i}\end{array}\right]$, the following equation can be derived under a "slight" motion hypothesis: 


$$
A=E \ddot{Q} .
$$

Accordingly, $\ddot{Q}$ can be computed using the least-squares method:

$$
\ddot{Q}=\left(E^{T} E\right)^{-1} E^{T} A,
$$

where $E$ is the transpose matrix and $A$ denotes the acceleration vectors in three orthogonal directions for all the points.

$$
\begin{aligned}
& E=\left[\begin{array}{cccccc}
1 & 0 & 0 & 0 & z_{1} & -y_{1} \\
0 & 1 & 0 & -z_{1} & 0 & x_{1} \\
0 & 0 & 1 & y_{1} & -x_{1} & 0 \\
& & & \cdots & & \\
1 & 0 & 0 & 0 & z_{s} & -y_{s} \\
0 & 1 & 0 & -z_{s} & 0 & x_{s} \\
0 & 0 & 1 & y_{s} & -x_{s} & 0
\end{array}\right],
\end{aligned}
$$

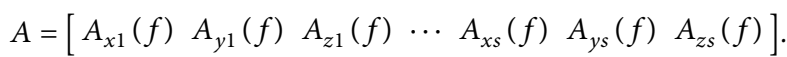

We can precisely extract the acceleration signal phases and amplitudes by exploiting the engine acceleration signals at the mounts and block by using discrete spectrum interpolation. The generalised force at the CG [17] is given by

$$
F(f)=\left[M-\frac{1}{\omega^{2}} K^{*}\right]\left(E^{T} E\right)^{-1} E^{T} A .
$$

At local coordinates, the mount complex stiffnesses in the three directions are given as

$$
\begin{aligned}
K^{*} & =K+j \omega c \\
& =K+j K^{\prime},
\end{aligned}
$$

where $j=\sqrt{-1}$ and $K^{\prime}=\omega C=2 \pi f C$ are the loss stiffnesses.

The phase difference of the gas-pressure torque for each cylinder of an $i$-cylinder engine is $4 \pi / i$, which varies with the engine ignition order. The highest amplitude of the first harmonic order $k=i / 2$ appears in the resultant torque spectrum. The primary harmonic orders of a 4-cylinder 4stroke 4-cylinder 4-cylinder engine include $k=i / 2, i, 3 i / 2$. Figure 2 shows the phase angle graphs for a typical 4-cylinder 4-stroke 4-cylinder 4-cylinder engine, the firing order of which is1-3-4-2.

Considering the same contribution by each cylinder to the engine torque, primary orders in the torque's structure are merely present due to gas forces, which are multiples of half the cylinder quantity for the 4-cylinder 4-stroke engine (Figure 2). Besides, the primary harmonic orders for the torque are the $2 \mathrm{nd}, 4$ th, and 6 th orders, when the engine runs as usual. Primary harmonic orders change in the event of a fault.

2.2. Interpolation Method for Discrete Spectrum. Equation (9) requires accurate extraction of the amplitudes, frequencies, and phases of the engine mounts and block. However, actual test signals contain noise. In discrete spectral analyses, no- integer period sampling leads to erroneous amplitude, frequency, and phase [18-21]. Conventional FFT extracts a highly errored generalised force, especially for the phase. Before the correction, the first harmonic of the acceleration shows similar errors to those for absolute phases. Thus, attaining accurate relative phases between acceleration responses leads to a decrease in the error estimates of the generalised force. However, in the 2nd harmonic, phase errors vary, provided the failure of maximum spectral lines is within different frequencies, and alterations are present in the relative phases [17]. The interpolation method is used to estimate frequency bias based on the amplitude ratio of the first two maximum spectral lines. Consider $x(t)$ as a single sequence of the harmonic signal and its amplitude, frequency, and phase as $f_{0}, A$, and $\theta_{0}$, respectively. Then,

$$
x(t)=A \cos \left(2 \pi f_{0} t+\theta_{0}\right) .
$$

Through equispaced sampling using $N$ sampling points at sampling frequency $f_{0}$, we get a new sampling sequence:

$$
x(n)=A \cos \left(2 \pi f_{0} \frac{n}{N}+\theta_{0}\right) .
$$

Applying Fourier transformation to equation (12), it becomes

$$
X_{w}(k)=\frac{1}{N} \sum_{k=0}^{N-1} w(n) x(n) e^{-j 2 \pi n k / N},
$$

where $w(n)$ is the window function.

After correction, the amplitude and phase are given by

$$
\begin{aligned}
& \widehat{A}=\frac{X_{w}(k)}{W\left(\nabla f^{1}\right)}, \\
& \widehat{\theta}_{0}=\arctan \left(\frac{I_{k}}{R_{k}}\right)+\pi \nabla f^{1},
\end{aligned}
$$

where $X_{w}(k)$ is the amplitude of the maximum spectral line of the discrete spectrum of harmonic signals, that is, the greatest amplitude in the major lobe, and $W\left(\nabla f^{1}\right)$ is the spectral mode function.

The rectangle window is expressed as

$$
W\left(\nabla f^{1}\right)=\frac{\sin \left(\pi \nabla f^{1}\right)}{\pi \nabla f^{1}} .
$$

The Hanning window is expressed as

$$
W\left(\nabla f^{1}\right)=\frac{\sin c\left(\nabla f^{1}\right)}{1-\nabla f^{1}} .
$$

Highly accurate amplitudes can be attained even at a considerably low sound-to-noise ratio (SNR) of $-2.33 \mathrm{~dB}$. The maximum error rates for the rectangle and Hanning windows are $4.5 \%$ and $6 \%$, respectively. The phase precision for the Hanning window is $\left\langle 10^{\circ}\right.$; however, due to an improper interpolation direction in the interpolation technique that is multiplied with the rectangular window when $\mathrm{SNR}=2.33 \mathrm{~dB}$ and $\nabla f_{1} \leq 0.25$, the resulting error is 

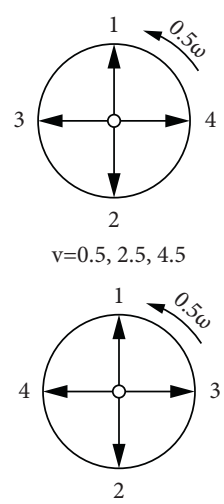

$\mathrm{v}=1.5,3.5,6.5$ $\mathrm{v}=0.5,2.5,4.5$

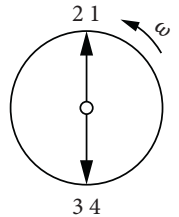

$\mathrm{v}=1,3,5$

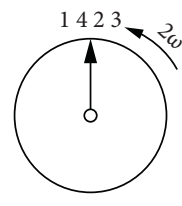

$\mathrm{v}=2,4,6$
Figure 2: Phase angle graphs of gas-pressure torques for the 4cylinder 4-stroke engine.

relatively large. The Hanning window is twice in width as the rectangle window, leading to a significant reduction in the possibility of interpolation direction error, and there is no arch exception [18]. Hence, we applied Hanning windowed interpolation discrete spectrum in order to extract the phase, amplitude, and frequency of the engine acceleration signals.

\section{Camshaft Loosening Diagnosis Based on Generalised Force Recognition}

3.1. Simulation Models. The engine valve mechanism comprises a cam, a lifter, valves, valve springs, and a camshaft. Each component has a certain mass and elasticity to produce an elastic deformation during motion. The valve train produces high acceleration when running at a high speed and is subject to a specific dynamic load. In the dynamic analysis, the components of the valve mechanism are simplified into an equivalent lumped mass and an equivalent spring, respectively. Thus, the lumped mass-spring vibration model can be applied to perform the dynamic analysis of the valve mechanism. The dynamic model of the valve train has been designed based on AVL Excite Timing Drive and includes double overhead camshafts for a 4-cylinder 4-stroke engine. The component unit of the valve train contains parts such as a valve stem, valve seat, camshaft bearing, cam, lifter, and spring and phase units, in sync with the composition of the valve mechanism. Figure 3 presents the logic schematic diagram of the component connections of the valve train.

We performed a high-quality FEA using AVL Excite and multibody dynamics modelling for an in-line 4-cylinder 4stroke engine installed with mounts (Figure 4). First, an engine crankshaft and powertrain finite element model is established. The finite element has an enormous degree of freedom; thus, its matrix is simplified using MSC Nastran to improve the computational efficiency. Next, the coupled multibody dynamics model is set up based on the AVL Excite. The excitation force includes the force of a gas explosion and the inertial forces of reciprocation and rotation. All excitation forces are included in our model to ensure that the vibration is similar to that of an actual engine. The explosion pressure for each cylinder is set based on the cylinder pressure plot. The cylinder pressure curve of an engine running at a speed of $2000 \mathrm{rpm}$ at full load condition is shown in Figure 5. The piston load of each cylinder at regular intervals, the pressure load of each cylinder wall, and the load torque at the flywheel output are generated automatically by the software as per the firing order. The same is applied to the corresponding nodes. The valve mechanism force can be considered an extended load of the powertrain, which contains valve seat force, valve spring force, and camshaft support force. The other force is the piston striking force produced due to the gap between the piston and the cylinder liner.

For determining the valve train excitation force, we used the valve train model based on the Excite Timing Drive and loaded the calculated force into the Power Unit model. The data connection interface is preferred for both models. The piston striking force is calculated using excited Piston and Rings, and the dynamic striking force of five nodes is distributed in the height direction of the main and vice thrust side of the cylinder liner. The calculated piston striking force is also considered an extended force loaded into the corresponding nodes of the powertrain model.

Table 1 presents the CG of the power assembly and the mount locations. Table 2 presents the power assembly's inertia parameters excluding those of the piston, connecting rod, and crankshaft. The three mounts have a stiffness of $3 \times 10^{5} \mathrm{~N} / \mathrm{m}$ and damping of $200 \mathrm{~N} \mathrm{~s} / \mathrm{m}$.

3.2. Camshaft Bearing Loosening Fault Setting. The simulation of loosening bearing faults is created by removing the SRBS unit from the model, provided that fixation of intake and exhaust camshafts at the cylinder head is achieved using five sliding bearings. No effect is exerted by the loosening bearing on the pressing camshaft. In the present simulation, the bearing of the intake camshaft of cylinder-3 (SRBS int4) is removed so that bearing- 4 has a zero acting force throughout the work cycle, and the dynamic driving force of cylinder-3's intake and exhaust valves is transmitted to the cylinder through the adjacent bearings 3 and 5 only. Figures 6 and 7 display the bearing forces of bearing 3 and 5, respectively, at 2,000 rpm under normal and faulty scenarios. When the bearing of cylinder-3 loosens, the supporting force of the adjacent bearing changes to a large extent.

The excitation force of the valve mechanism is taken as the input force for the engine powertrain. The acting force of the camshaft on the cylinder head is mainly transmitted through the bearing. The valve train excitation force is calculated based on the valve train model, followed by introducing force into the Power Unit model through the loading points as shown in Figure 8.

3.3. Camshaft Loose Diagnosis. The loosening of the camshaft bearing changes the generalised force at the CG. Thus, the fault features of camshaft bearing loosening can be determined by calculating the generalised force based on the vibration signals of the engine system. The Hanning windowed interpolation, as mentioned in Section 2.2, is used for the precise phase, frequency, and amplitude extraction of the 


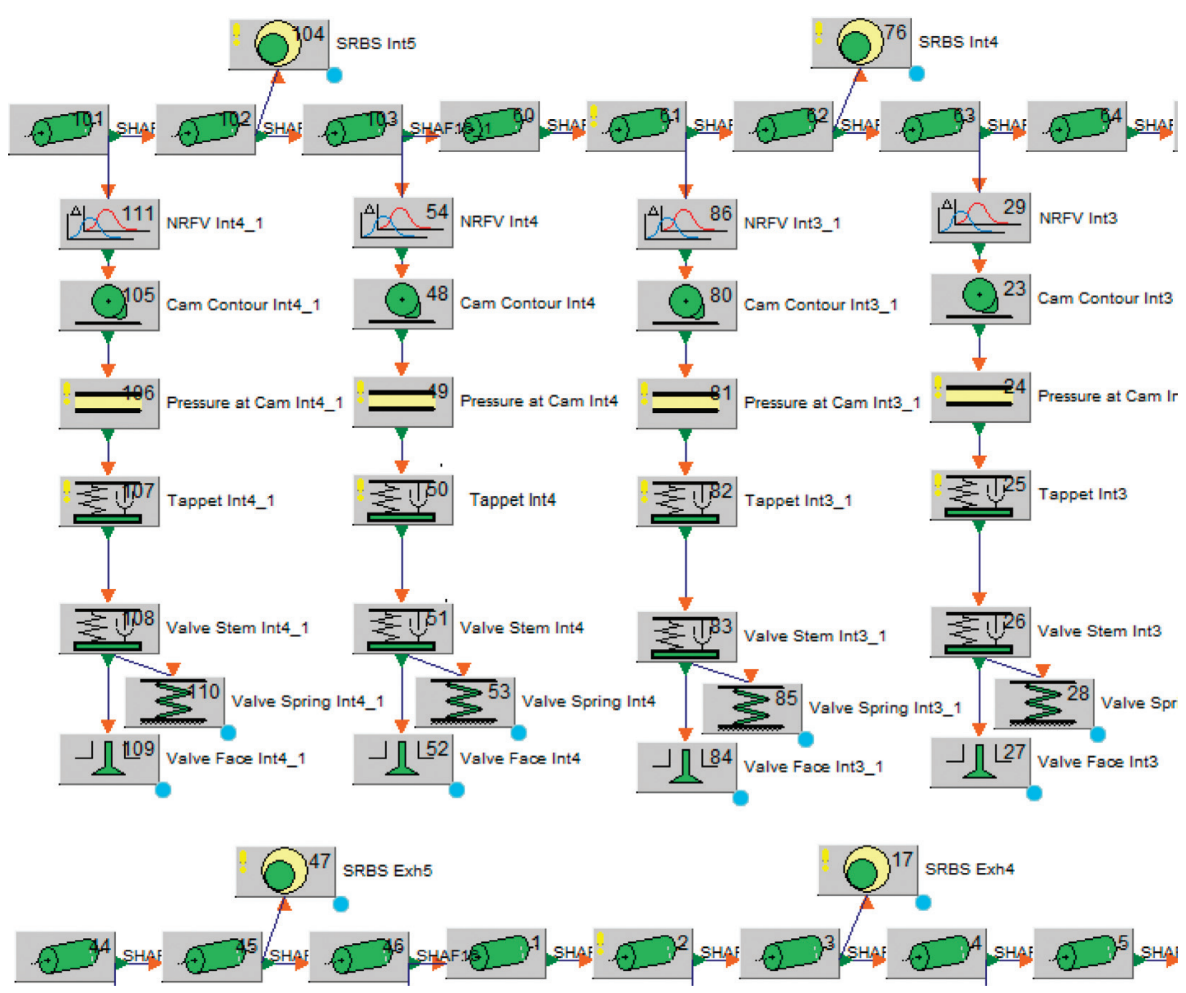

Figure 3: Valve train logic of component connections.

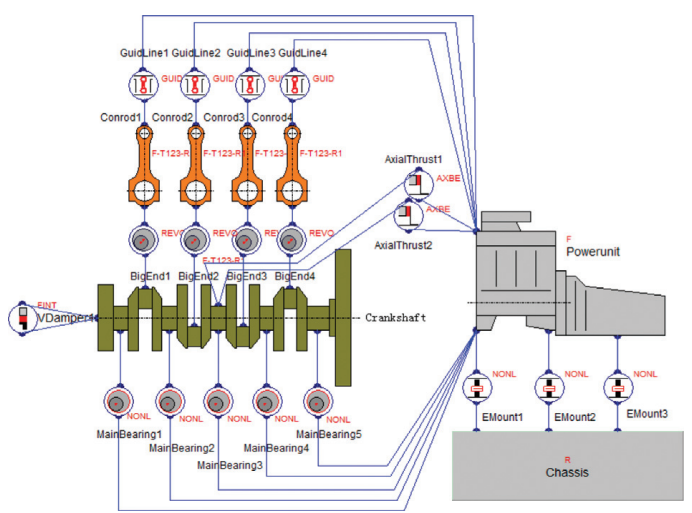

Figure 4: Engine logic of component connections.

three engine acceleration signals at the cylinder block, head, and mounts, respectively. The generalised force computation at the CG is determined using equation (9).

The engine has a firing order of 1-3-4-2. The frequency of the excitation signal of the 4-cylinder 4-stroke engine is twice that of the engine RPM. The vibration signals produced by the engine are the superposition of the fundamental frequency signal and its higher frequency signal, based on the harmonic signal characteristics. Under normal conditions, the primary harmonic orders of engine vibration signal are the $2 \mathrm{nd}$, 4 th, and 6 th orders, respectively, whereas the 0.5 th order is nearly zero. The highest amplitude of the 2nd harmonic appears on the resultant torque spectrum. Additionally, the signal of the engine block vibration conforms to the same law.
The vertically generalised force, identified under normal conditions, is shown in Figure 9. The amplitude of the 2 nd harmonic is the highest, whereas the amplitude of the 0.5 th harmonic is closer to zero, which is consistent with the theoretical analysis. Camshaft loosening was induced in cylinder-3 by removing the intake camshaft bearing SRBS int4 (Figure 1). In this case, a large impact is observed after each alternate revolution by the engine, and its frequency is half of the engine RPM. The loosening of the camshaft leads to the formation of another excitation force cycle, and the crankshaft rotates twice per cycle. It also leads to an increase in the amplitude of the 0.5 th harmonic and its multiples increases considerably (Figure 10). 


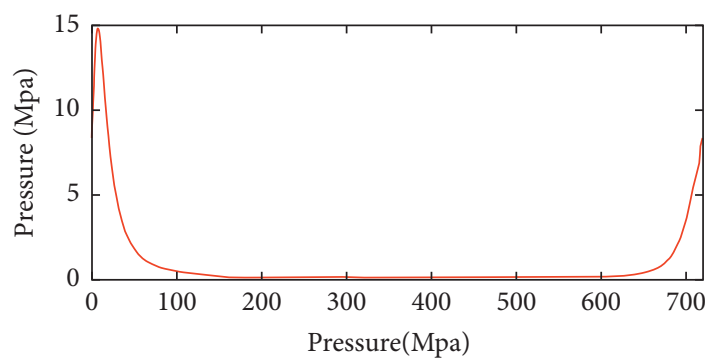

Figure 5: Pressure curve of the cylinder at $2000 \mathrm{rpm}$.

TABLE 1: Engine CG and mount positions.

\begin{tabular}{lccc}
\hline & $X$ & $Y$ & $Z$ \\
\hline Engine c.g. & $139.3 \mathrm{~mm}$ & $9.8 \mathrm{~mm}$ & $91.9 \mathrm{~mm}$ \\
Mount 1 & 0 & $285.2 \mathrm{~mm}$ & $122 \mathrm{~mm}$ \\
Mount 2 & $73 \mathrm{~mm}$ & $-254 \mathrm{~mm}$ & $-45.5 \mathrm{~mm}$ \\
Mount 3 & $554.5 \mathrm{~mm}$ & $-80.4 \mathrm{~mm}$ & $-148.1 \mathrm{~mm}$ \\
\hline
\end{tabular}

TABLE 2: Engine inertia variables.

\begin{tabular}{|c|c|c|c|c|c|c|}
\hline Mass & & ment of iner & & & oduct of inert & \\
\hline $\begin{array}{l}m \\
111 \mathrm{~kg}\end{array}$ & $\begin{array}{c}J_{x x} \\
4.13 \mathrm{~kg} \mathrm{~m}^{2}\end{array}$ & $\begin{array}{c}J_{y y} \\
9.67 \mathrm{~kg} \mathrm{~m}^{2}\end{array}$ & $\begin{array}{c}J_{z z} \\
8.13 \mathrm{~kg} \mathrm{~m}^{2}\end{array}$ & $\begin{array}{c}J_{x y} \\
0.16 \mathrm{~kg} \mathrm{~m}^{2}\end{array}$ & $\begin{array}{c}J_{y z} \\
-0.16 \mathrm{~kg} \mathrm{~m}^{2}\end{array}$ & $\begin{array}{c}J_{x z} \\
1.12 \mathrm{~kg} \mathrm{~m}^{2}\end{array}$ \\
\hline
\end{tabular}

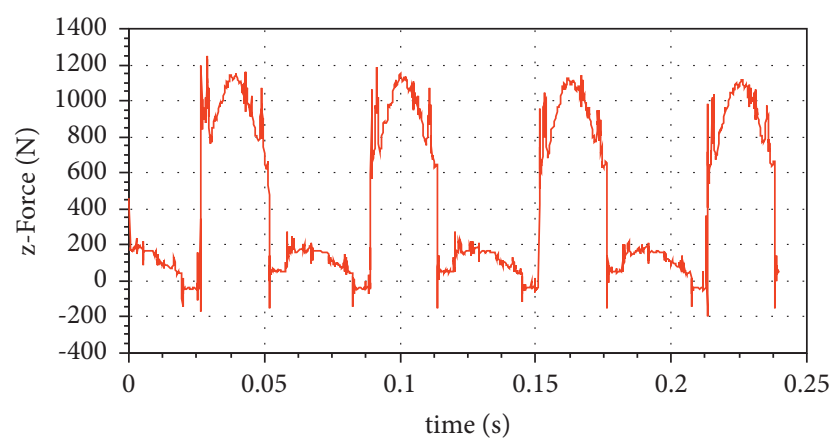

— Camshaft normal Force (N)

Figure 6: Camshaft force under normal condition.

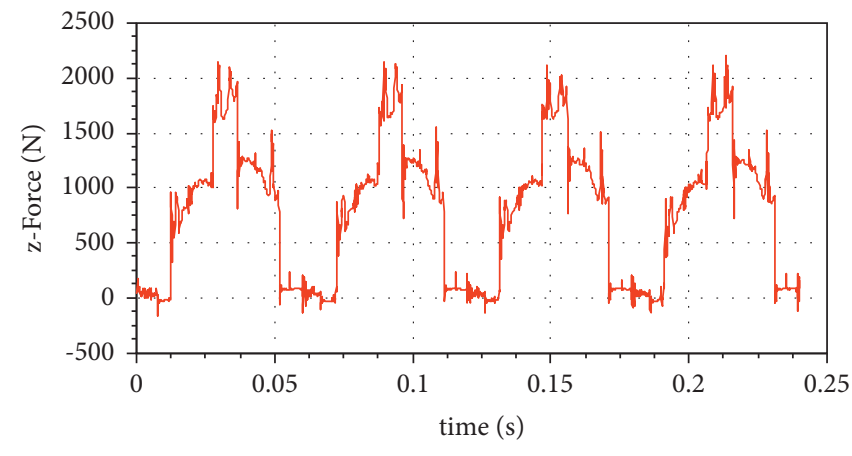

- Camshaft loose Force (N)

Figure 7: Camshaft force in faulty state. 


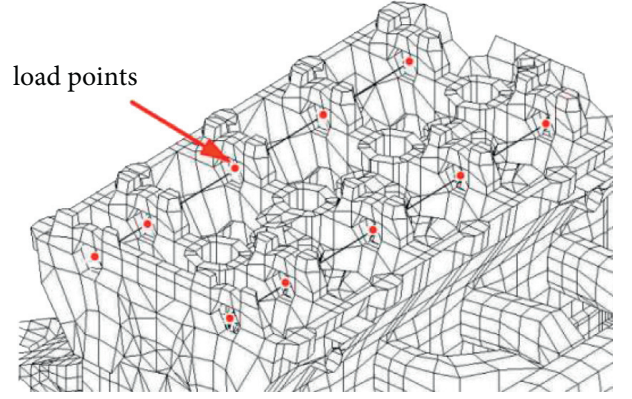

FIGURE 8: Bearing force load point.

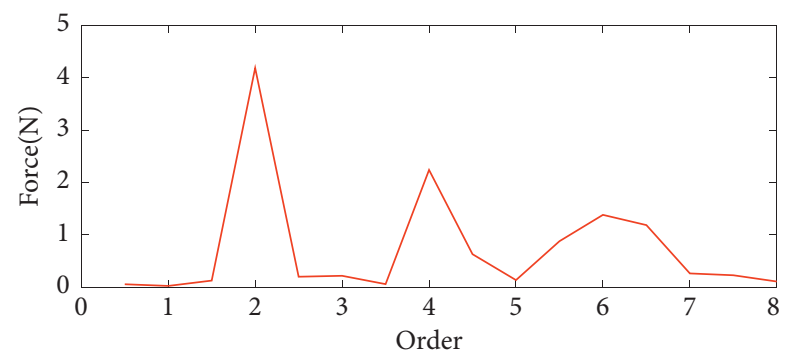

FIgURE 9: Generalised vertical force identified at the engine CG.

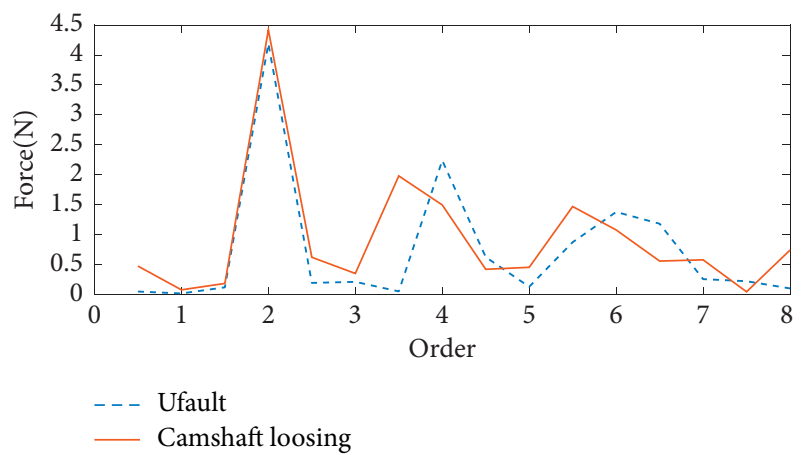

FIGURE 10: Vertical generalised force of camshaft loosing.

\section{Conclusions}

This study proposed a novel technique for detecting camshaft loosening, which was used for analysing the order of the harmonics of the generalised force at the CG. Precise phase, frequency, and amplitude extraction were conducted using the Hanning windowed interpolation, thereby achieving the generalised force accurately at the CG.

The proposed method is effective in diagnosing the fault induced due to camshaft loosening as demonstrated by the simulation results based on the AVL software. The loosening of the camshaft changes the generalised force at the CG. The amplitude of the 0.5 th harmonic and its multiples increase sharply because the crankshaft rotates twice per cycle. The 0.5 th harmonic and its multiples increase when the rate of change reaches half of the engine speed.

\section{Data Availability}

No data were used to support this study.

\section{Conflicts of Interest}

The authors declare that there are no conflicts of interest regarding the publication.

\section{Acknowledgments}

This work was supported under Grant nos. 51405272 and 51505258 from the National Natural Science Foundation of China and partially supported by the Natural Science Foundation of Shandong Province (ZR2020ME126), the Youth Science and Technology Project of Shandong Provincial Colleges and Universities (Grant no. 2019KJB019), Shandong Provincial Major Scientific and Technological Innovation Project (2019JZZY020617), Shandong Provincial Key Research and Development Program (2019GNC106071), and State Key Laboratory of Mechanical Behavior and System Safety of Traffic Engineering Structures, China (Grant no. 1903).

\section{References}

[1] H. L. Bi, "Wear analysis of a camshaft and roller tappet of an engine," Master's thesis, Shandong University, Jinan, China, 2017.

[2] W. Y. Zhi and X. L. Xu, "Failure analysis on fractured diesel engine camshafts," Journal of Failure Analysis and Prevention, vol. 9, no. 1, pp. 39-42, 2009.

[3] X. Duan, J. Long, C. Li, and D. Cabrera, "Intelligent fault diagnosis of 3D printers based on reservoir computing," International Journal of Performability Engineering, vol. 15, no. 12, pp. 3171-3178, 2019.

[4] C. Y. Du and F. F. Yu, "Analysis of engine camshaft bearing loosening fault based-on model simulation and vibration signal," Advanced Materials Research, vol. 694-697, pp. 896-900, 2013.

[5] X. Y. Li, J. W. Guo, X. J. Jia, S. H. Zhang, and Z. Y. Liu, "Intelligent fault diagnosis of delta 3D printers using attitude sensors based on extreme learning machines," International Journal of Performability Engineering, vol. 15, no. 12, pp. 3196-3208, 2019.

[6] S. Zhang, M. Wang, C. Du, and E. Estupinan, "Local and global SR for bearing sensor-based vibration signal classification," International Journal of Performability Engineering, vol. 15, no. 10, pp. 2657-2669, 2019.

[7] A. Kumar, C. P. Gandhi, Y. Zhou et al., "Improved CNN for the diagnosis of engine defects of 2-wheeler vehicle using wavelet synchro-squeezed transform (WSST)," KnowledgeBased Systems, vol. 208, Article ID 106453, 2020.

[8] D. Ting, H. G. Wang, and S. Lei, "Study of camshaft grinders faults prediction based on RBF neural network," Applied Mechanics and Materials, vol. 141, pp. 519-523, 2012.

[9] Y. G. Shi, X. Y. Liu, and J. W. Xiang, "FEM simulation-based generative adversarial networks to detect bearing faults," IEEE Transactions on Industrial Informatics, vol. 16, no. 7, pp. 4961-4971, 2020.

[10] Z. M. Bulatovic, M. S. Stavljanin, and M. V. Tomic, "Measurement and analysis of angular velocity variations of twelvecylinder diesel engine camshaft," Mechanical Systems and Signal Processing, vol. 25, no. 8, pp. 3041-3061, 2011.

[11] X. Jiang, C. Shen, J. Shi, and Z. Zhu, "Initial center frequencyguided VMD for fault diagnosis of rotating machines," Journal of Sound and Vibration, vol. 435, pp. 36-55, 2018. 
[12] Y. Wang, Z. He, and Y. Zi, "A demodulation method based on improved local mean decomposition and its application in rub-impact fault diagnosis," Measurement Science and Technology, vol. 20, no. 2, Article ID 025704, 2009.

[13] J. Guo, W. P. Zhang, and X. Y. Zhang, "Modeling and analysis of the transient vibration of camshaft in multi-cylinder diesel engine," Advances in Mechanical Engineering, vol. 7, no. 11, pp. 1-14, 2015.

[14] A. Kumar, C. P. Gandhi, Y. Zhou, R. Kumar, and J. Xiang, "Latest developments in gear defect diagnosis and prognosis: a review," Measurement, vol. 158, Article ID 107735, 2020.

[15] C. Xu, S. Li, F. Cao, and X. Qiu, "Misfire detection based on generalized force identification at the engine centre of gravity," IEEE Access, vol. 7, pp. 165039-165047, 2019.

[16] C. M. Wang, Vehicle Engine Dynamics, National Defense Industry Press, Beijing, China, 1990.

[17] C. Xu and F. Cao, "Engine excitation force identification on the basis of discrete spectrum correction," Mathematical Problems in Engineering, vol. 2015, Article ID 175257, 11 pages, 2015.

[18] X. Ming and D. Kang, "Corrections for frequency, amplitude and phase in a fast fourier transform of a harmonic signal," Mechanical Systems and Signal Processing, vol. 10, no. 2, pp. 211-221, 1996.

[19] C. Y. Xu, K. Ding, H. B. Lin, and Z. J. Yang, "Noise influence on amplitude and phase estimation accuracy by interpolation method for discrete spectrum," Journal of Vibration Engineering, vol. 24, no. 6, pp. 633-638, 2011.

[20] H. B. Lin and K. Ding, "Energy based signal parameter estimation method and a comparative study of different frequency estimators," Mechanical Systems and Signal Processing, vol. 25, no. 1, pp. 452-464, 2011.

[21] C. Y. Xu, K. Ding, Z. J. Yang, and H. B. Lin, "Influence of additive white gaussian noise on the interpolation method of discrete spectrum," Advanced Materials Research, vol. 383-390, pp. 2951-2957, 2012. 